\title{
Caracterização mecânica de painéis particulados de média densidade produzidos a partir de resíduos de madeira
}

Cristiane Karyn Carvalho Araujo criskaryn.carvalho@hotmail.com Universidade Tecnológica Federal do Paraná (UTFPR), Ponta Grossa, Paraná, Brasil

Cristiane Inácio de Campos cristiane@itapeva.unesp.br Universidade Estadual Paulista (UNESP), Itapeva, São Paulo, Brasil

Sâmique Kyene de Carvalho Aráujo Camargo

samiquekyene@hotmail.com Universidade Estadual Paulista (UNESP) Guaratinguetá, São Paulo, Brasil

Bruno Silva Camargo bs.camargo@hotmail.com Uns.camargo@hotmail.com Paraná (UTFPR), Ponta Grossa, Paraná, Brasil

\begin{abstract}
RESUMO
Nos últimos anos o setor florestal vem se destacando por inovações tecnológicas e criações de novos materiais e produtos. Com isso, a geração de resíduos referente ao processamento da madeira ainda é um grande problema para o segmento, pois embora as novas tecnologias apresentem maquinários mais modernos, o volume gerado ainda é bastante significativo. Com a expressiva geração de resíduos nas indústrias de base florestal, surge à necessidade de utilizar tais resíduos de modo a agregar valor aos mesmos e minimizar o impacto ambiental proporcionado. Neste segmento de novos materiais à base de madeira a partir de resíduos como fonte de matéria-prima pode-se destacar os painéis, principalmente, particulados (MDP) e de fibras (MDF). O MDP faz parte da nova geração de Painéis de Partículas de Média Densidade, onde suas características são superiores e totalmente distintas dos painéis de madeira aglomerada de antigamente. A partir desta disposição do mercado madeireiro definiu-se o objetivo do presente estudo, que consistiu em produzir o painel de madeira a partir de resíduos de Eucaliptus. sp. e avaliar o comportamento da resistência mecânica frente à variação da porcentagem granulométrica das partículas da camada interna do painel, produzidos a partir de resíduos. A caracterização mecânica foi embasada no documento normativo da Associação Brasileira de Normas Técnicas ABNT-NBR 14810-2/2013. Os resultados obtidos foram analisados estatisticamente e, em seguida comparados com a norma brasileira bem como, trabalhos encontrados na literatura.
\end{abstract}

PALAVRAS-CHAVE: MDP. Caracterização. Resíduos. 
Nos últimos anos o setor florestal vem se destacando, de acordo com a Indústria Brasileira de Árvores (2017) o Brasil é líder mundial em produtividade de madeira, e possui como objetivo a intensificação da produção para atender a crescente demanda pelas novas aplicações que a madeira permite, sempre com o comprometimento de trabalho e manejo sustentável das florestas, que exercem papel relevante na proteção e conservação dos ecossistemas.

Considerando o aumento na demanda deste setor, novas inovações tecnológicas e criações de materiais e produtos surgiram para suprir esta necessidade. Devido a estes fatores nota-se que a geração de resíduos referente ao processamento da madeira também cresceu, em volume significativo.

Com a expressiva geração de resíduos no setor florestal, surge à necessidade de utilizar tais resíduos de modo a agregar valor e minimizar o impacto ambiental proporcionado. Com base nos aspectos apresentados, surgiu à proposta de melhor aproveitamento da matéria-prima a partir de processos que incorporem conceitos de gestão ambiental, logística reversa e os princípios de economia circular, que trazem benefícios ambientais e vantagens econômicas as empresas (WEBER, 2011).

Dentre estas aplicações para atender a proposta de melhor aproveitamento da madeira, destacam-se os painéis de madeira, principalmente os de madeira reconstituída, que apresentam um grande dinamismo, refletido pela inequívoca competitividade do setor florestal, qualidade e grande aceitação do produto no mercado nacional (BNDES, 2008), que desde 2009 vem apresentando um crescimento de $3,7 \%$ ao ano.

Os painéis de madeira reconstituída são produzidos a partir de diferentes etapas de desagregação da madeira e compactados por adição de adesivos sintéticos sob ação de pressão e temperatura. A partir desta disponibilidade na produção de painéis de madeira, vê-se a oportunidade de inserção de resíduos em sua composição. Segundo Souza et. al., (2018) os resíduos de madeira podem retornar ao processo produtivo, a fim de agregar valor nos compósitos, visando trazer vantagens econômicas e ambientais para as indústrias, ao invés do descarte inadequado.

Os resíduos de madeira apresentam potencial econômico viável, pouco aceito pelas indústrias. Porém, a alternativa de aproveitamento desses resíduos para o desenvolvimento de novos produtos lignocelulósicos tem a finalidade de os mesmos formarem a matriz principal do novo produto, sempre atentando a qualidade final do produto gerado. Neste segmento de novos materiais à base de madeira a partir de resíduos como fonte de matéria-prima pode-se destacar os painéis de MDP e MDF.

Os painéis particulados e de fibras produzidos a partir de resíduos de madeira apresentam possibilidade de aplicações em diversos segmentos, podendo-se destacar a indústria moveleira e a construção. No entanto, o desempenho dos produtos a partir da reutilização de matérias-primas pode trazer algumas limitações ao produto final, a qualidade do novo produto nem sempre terá as propriedades do produto produzido com matéria-prima 
proveniente de florestas plantadas, onde o controle e qualidade da madeira são maiores e melhores para o desempenho final do produto.

Com base nos aspectos apresentados anteriormente é que surge a proposta deste estudo que foi produzir painel particulado de madeira composto por três camadas a partir de resíduos de eucalipto gerados no processamento mecânico da madeira, incentivando a produção sustentável, variando também a granulometria da matéria-prima constituinte da camada intermediária de modo a avaliar o comportamento mecânico deste produto frente a modificações.

\section{REFERENCIAL TEÓRICO}

Os produtos de madeira vêm se destacando por sua grande versatilidade, com isso vários produtos surgem para atender a grande demanda, principalmente, no setor moveleiro, assim como na construção civil. Com isso desenvolveu-se produtos florestais, tais como os painéis. Tendo em vista o melhor aproveitamento da madeira proporcionado na produção dos painéis, evitando excesso de desperdício de matéria-prima, os mesmos se apresentam como alternativa ao mercado.

No segmento dos painéis à base de madeira podem-se destacar os painéis: MDP (painéis de média densidade), MDF (painéis de fibras de média densidade), de madeira compensada, OSB (painéis de lascas orientadas), e HDF (painéis de fibras de alta densidade).

Segundo Youngquist (1999), os painéis podem ser divididos em três principais grupos, sendo:

- Lâminas - chapa de madeira compensada (plywood), chapa de madeira sarrafeada, e LVL (laminated veneer lumber);

- Partículas - chapa de madeira aglomerada (particleboard), chapa de "tiras" de madeira não orientadas (WB - waferboard), chapa de "tiras" de madeira orientadas (OSB - oriented strand board), OSL (oriented strand lumber) e LSL (laminated strand lumber);

- Fibras - chapa de fibra isolante (IC), chapa dura de fibra e chapa de alta densidade (HD - hardboard) e chapa de fibra de média densidade (MDF).

Sellers (2000) destaca que a demanda por produtos compósitos de madeira, tais como compensado, chapa de lascas orientadas, chapa dura, aglomerado, chapa de fibra de média densidade e chapas compostas de lâminas recentemente cresceu substancialmente em todo o mundo.

\section{Painéis de MDP (MEDIUM DENSITY PARTICICLEBOARD)}

O MDP é um painel de madeira reconstituída, assim como o MDF e o HDF (IBÁ, 2015). MDP é a abreviação de Medium Density Particleboard, ou seja, chapa de partículas de média densidade.

O MDP é o resultado do uso intensivo de tecnologia de prensas contínuas, de modernas tecnologias de classificadores de partículas, fazendo uso de resinas de última geração e madeira de florestas plantadas. O MDP faz parte da nova 
geração de Painéis de Partículas de Média Densidade, onde suas características são superiores e totalmente distintas dos painéis de madeira aglomerada de antigamente (REMADE, 2006).

No painel de MDP, as partículas são posicionadas de forma diferenciada, as de maiores dimensões dispostas ao centro e as de menores nas superfícies externas, compondo assim as três camadas do painel. As partículas são aglutinadas com resina sintética, e compactadas através de pressão e temperatura em prensa contínua (IBÁ, 2015). Os painéis são produzidos com madeira de reflorestamento, tais como, Pinus e Eucalyptus.

\section{MATÉRIA- PRIMA}

Segundo Iwakiri (2005), os insumos básicos usados na produção de chapa de partículas de média densidade (MDP) são: madeira, resina e catalisador. Outros componentes também podem ser usados na produção dos painéis, sendo emulsão de parafina, retardantes de fogo, preservantes contra fungos e insetos. Estes aditivos são adicionados para melhorar as propriedades do painel para determinadas aplicações, ou seja, aplicações específicas.

\section{Madeira}

A madeira utilizada na fabricação de painéis como matéria-prima é proveniente de reflorestamento, tais como, folhosas ou coníferas, sendo a escolha feita devido às características que se deseja para o painel, também a partir da região e disponibilidade de matéria-prima nesta localidade. No Brasil as madeiras mais utilizadas são os gêneros de eucaliptos e Pinus. A Figura 1 demonstra a distribuição das plantações de eucaliptos e Pinus por região.

Figura1: Área Florestal Plantada com Pinus e Eucalipto no Brasil (2016).

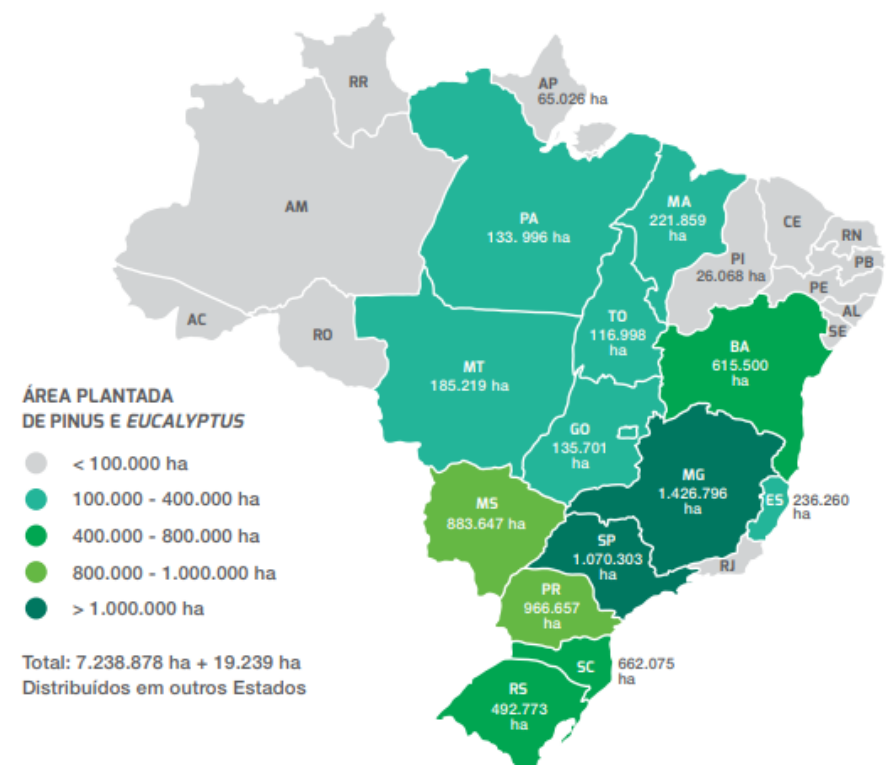

Fonte: APRE (2017-2018). 
Atualmente as empresas brasileiras utilizam na maioria das vezes, madeira em forma de toretes, sendo estes de espécies de madeira de rápido crescimento, tais como do gênero de Pinus e Eucalyptus. Os toretes utilizados apresentam pequenos diâmetros que variam de $3 \mathrm{~cm}$ a $5 \mathrm{~cm}$ (mínimo) até $25 \mathrm{~cm}$ (máximo), são provenientes de plantios exclusivos para estes fins, também de desbastes, resíduos de exploração e árvores caídas.

A partir dos toretes é possível obter partículas com comprimento que variam de $0,5 \mathrm{~m}$ a $0,6 \mathrm{~m}$, sendo mais comum a utilização de toretes de $2 \mathrm{~m}$ a 2,5 $\mathrm{m}$. Para a escolha correta da madeira a ser utilizada deve-se levar em consideração as propriedades, tal como a densidade, que é uma das mais importantes, sendo necessário o acompanhamento após a chegada à indústria, pois apresenta relação direta no processo, sendo a compressibilidade e o teor e adesivo a ser empregado, para que se alcance qualidade na adesão interna do painel (PIERRE, 2010).

\section{Adesivos utilizados na produção de painéis}

Na produção de painéis de madeira um dos principais componentes é a resina, utilizada como aglutinantes das partículas de madeira. Destacam-se como resinas sintéticas mais usadas nas indústrias de painéis, a Uréia-Formaldeído sendo a mais predominante, seguido de Fenol-Formaldeído e MelaminaFormaldeído (MALONEY, 1993). Sendo definidas:

- Uréia-formaldeído: resina produzida em solução aquosa contendo sólidos entre $60 \%$ e $70 \%$, ou em forma de pó. Sua coloração é branca leitosa, seu pH se encontra na faixa de 7,4 a 7,8 e viscosidade na faixa de 400 a 1000 cP. Apresenta custo inferior em relação às outras resinas. Sua desvantagem é a baixa resistência à umidade, utilizada então em locais onde a umidade é a mínima possível, uso interno (IWAKIRI, 2005).

- Melamina-formaldeído: Utilizada como uso intermediário, pois possui maior resistência à umidade em relação à uréia-formaldeído. Sua coloração também é branca leitosa, podendo ser utilizada como fortificante se quando misturada com uréia-formaldeído. $\mathrm{O}$ pH encontra-se em torno de 9 e a temperatura de cura entre $65^{\circ} \mathrm{C}$ a $1300^{\circ} \mathrm{C}$ (IWAKIRI, 2005). Quanto maior a temperatura, mais acelerado é o aumento da viscosidade da resina, assim, as resinas melamínicas têm um período de estabilidade mais baixo, e consequentemente o tempo de armazenagem mais reduzido quando comparado com a resina puramente de uréia (ALMEIDA, 2006).

- Fenol-formaldeído: Classificado para uso exterior, pois possui alta resistência à umidade. Sua coloração é marrom avermelhada, seu teor de sólidos encontra-se entre 48 e 51\%, pH variando de 11 a 13, e viscosidade entre 300 e $600 \mathrm{cP}$ e sua temperatura de cura na faixa de 130 a 150 ㅇ. $\mathrm{C}$. A sua aplicação destina-se principalmente à produção de compensados à prova d'água, painéis de fibras (duras), painéis aglomerados e estruturais "waferboard" e "OSB" (IWAKIRI, 2005). 
A resistência mecânica de uma chapa é afetada pela geometria das partículas, tais como nos testes de flexão estática, tração paralela e perpendicular à superfície da chapa. As partículas que apresentam comprimentos muito grandes geram problemas na secagem, aplicação de adesivo, e formação do colchão, pois se tornam volumosas, entopem equipamentos, dificultam o transporte do material, no entanto, o painel com essas partículas apresentam propriedades melhores. Segundo Gonçalves et al. (2000), as partículas são classificados quanto a sua geometria como:

- Cavacos: são partículas longas e espessas, seus tamanhos variam de 5 a 50 $\mathrm{mm}$, podendo ser com ou sem casca, obtidas por picadores no corte de madeiras descartadas.

- Serragem: são partículas curtas e finas, obtidas pelo corte de toras e retirada de costaneiras com dimensões entre 0,5 e $2,5 \mathrm{~mm}$.

- Maravalha: são denominadas como partículas longas e finas, variando a largura, obtidas pelo fresamento de peças de madeira e que apresentam dimensões maiores que $2,5 \mathrm{~mm}$.

\section{RAZÃO DE ESBELTEZ}

As partículas que apresentam maior comprimento são as melhores para a qualidade do painel, no entanto, as partículas de menor comprimento apresentam melhor qualidade superficial, dureza e melhores ligações internas (IRLE, 2012).

Segundo IWAKIRI (2005), a razão de esbeltez é a relação entre o comprimento e espessura das partículas, e possui grande influência na qualidade do painel.

A razão de esbeltez elevada proporciona ao painel menor absorção de água, inchamento em espessura e menor expansão linear, levando ao painel obter melhor estabilidade dimensional.

A geometria das partículas apresenta grande influência nas propriedades finais do painel, juntamente com as espécies da madeira, quantidade de adesivos e aditivos.

As partículas menores são usadas nas camadas superiores e inferiores do painel de partículas, onde as mesmas são aglutinas com resinas especiais. Essas promovem uma influência significativa na qualidade superficial dos mesmos, e as partículas maiores são usadas no centro do painel, onde as mesmas fornecem melhor resistência mecânica ao painel. Caso se mantenha a espessura da partícula, e se aumente o comprimento médio de sobreposição, conseqüentemente aumenta-se a relação entre o comprimento da partícula e a espessura (razão de esbeltez-s). Os valores de razão de esbeltez devem variar entre 120 a 200 para partículas das camadas externas, e de 60 para partículas da camada interna (IWAKIRI, 2005). 


\section{RAZÃO DE COMPACTAÇÃO}

A razão de compactação é a relação existente entre a massa específica do painel e da madeira utilizada. Esta razão apresenta o grau de densificação do material. A espécie de madeira para obter boa razão de compactação deve ser de baixa densidade. O valor adequado da razão de compactação para a produção de painel particulado encontra-se na faixa de 1.3 a 1.6 , por isso as espécies de menor densidade são as mais recomendadas. Os valores acima de 1.6 melhoram as propriedades de resistência, no entanto, o inchamento em espessura será maior, pelo fato da compressão existente sobre o painel na fase de prensagem (REMADE, 2006).

A densidade tem uma influência significativa sobre as propriedades das chapas e, também, no processo de produção. As chapas de partículas feitas com espécies de baixa densidade têm maior resistência à flexão, módulo de elasticidade, adesão interna e resistência à tração, já que apresentam maior qualidade de colagem. Para que as chapas produzidas com madeira de alta densidade possuam a mesma resistência que uma produzida com madeira de menor densidade deve-se aumentar obrigatoriamente a densidade da chapa, e isto implica em chapas com maior peso para o mesmo nível de resistência (IWAKIRI, 2005).

\section{MATERIAIS E MÉTODOS}

Nesta seção estão descritos os materiais utilizados, bem como a metodologia adotada para a produção dos painéis e realização dos ensaios mecânicos.

\section{MATERIAIS}

Os materiais utilizados para a produção dos painéis de MDP foram: resíduos de Eucalyptus sp., com teor de umidade de $3 \%$ provenientes de serraria e demais laboratórios da UNESP - Campus de Itapeva; Adesivo uréia-formaldeído, da marca Momentive, à base de água, com alta resistência a calor, teor de sólidos de aproximadamente $65 \%$, pH próximo ao neutro; Catalisador Cola Mite produzido pela Euroamerican com teor de sólidos de $20 \%$, composto por sulfato de amônia, utilizado para acelerar a cura do adesivo; Emulsão parafínica Humocer 100 A70 IS, produzida pela Isogama, com teor de sólidos catalisador; e Água.

\section{Equipamentos}

- $\quad$ Picador laboratorial (MARCONI-MA 683/3);

- Moinho tipo Willey (MARCONI- MA 680/5);

- $\quad$ Agitador de peneiras elétrico (BERTEL 50x50cm);

- Batedeira Industrial;

- $\quad$ Prensa hidráulica termo aquecida (Hidral-Mac PHH 80T). 


\section{MÉTODOS}

\section{PREPARAÇÃO DA MATÉRIA - PRIMA}

Os resíduos de madeira obtidos passaram pelo processo de desagregação em um picador, obtendo a partir desta, cavacos de diversas dimensões. Em seguida, os cavacos foram levados ao moinho tipo Willey e reduzidos a partículas de granulometrias variadas.

Para a produção do MDP as partículas devem ser classificadas conforme as dimensões desejadas, sendo assim, as partículas passaram pela classificação em um jogo de peneiras vibratórias $(50 \times 50 \mathrm{~cm}$ ), sendo o diâmetro das peneiras (9 e 16 mesh para as camadas internas e (35 e 60 mesh para as camadas externas).

\section{CONFECÇÃO DOS PAINÉIS DE MDP}

Posterior a formação dos colchões as partículas já classificadas, seguiram para a etapa de redução de teor de umidade, em estufa com circulação de ar forçado por aproximadamente 24 horas, com temperatura de $103^{\circ} \mathrm{C}(+-) 2^{\circ} \mathrm{C}$, até a obtenção de $3 \%$ de teor de umidade.

A proposta deste estudo foi avaliar somente o comportamento da resistência mecânica do painel produzido com resíduos de madeira, frente às variações na camada interna dos painéis, mantendo a camada externa inalterada para os três tratamentos (Tabela 1). As dimensões nominais dos painéis produzidos foram 45 $\mathrm{cm} \times 45 \mathrm{~cm} \times 12 \mathrm{~mm}$. A temperatura de prensagem foi de $150^{\circ} \mathrm{C}$, a pressão aplicada de $40 \mathrm{kgf} / \mathrm{cm} 2$, equivalente a 235 bar no manômetro da prensa e tempo de prensagem de 3 ciclos de 180 segundos, com 2 intervalos de 30 segundos para alívio de pressão. Para cada tratamento foram produzidos 3 painéis.

Tabela 1- Tratamentos e porcentagens de material

\begin{tabular}{|c|c|c|c|c|}
\hline \multirow[t]{2}{*}{ Tratamento } & \multicolumn{2}{|c|}{$\begin{array}{l}\text { Camada Externa } \\
\text { (Superior e Inferior) }\end{array}$} & \multicolumn{2}{|c|}{ Camada Interna } \\
\hline & 35 mesh & 60 mesh & 9mesh & 16 mesh \\
\hline Tratamento 1 & $70 \%$ & $30 \%$ & $30 \%$ & $70 \%$ \\
\hline Tratamento 2 & $70 \%$ & $30 \%$ & $50 \%$ & $50 \%$ \\
\hline Tratamento 3 & $70 \%$ & $30 \%$ & $70 \%$ & $30 \%$ \\
\hline
\end{tabular}

Para a formação total dos painéis foram utilizados 1600 gramas de partículas, 215 gramas de adesivo, 11 gramas de parafina, 7 gramas de catalisador e 16 gramas de água. Sendo decompostas da seguinte maneira (gramas):

Camada externa: Partículas (480); Adesivo (65); parafina (3); catalisador (2) e água (16)

Camada Interna: Partículas (1120); Adesivo (150); parafina (8); catalisador (5) e água (11), distribuindo as partículas conforme a porcentagem adotada em cada Tratamento.

Realizada a pesagem da quantidade de partículas para cada tratamento, as partículas foram encaminhadas ao processo de encolagem com adesivo 
comercial uréia-formaldeído, e adição de aditivos, em uma batedeira industrial, para homogeneização correta dos materiais. A encolagem ocorreu separadamente para cada camada (superior, central e inferior), devido à proporção de matéria-prima.

Em seguida, o material foi levado para a caixa formadora, sendo cada camada colocada em seu devido lugar, dimensionando e formando o colchão. Posteriormente o colchão passou pela pré-prensagem para a redução da espessura e, por fim levado para a prensa hidráulica Hidral-Mac, modelo PHH 80 $\mathrm{T}$.

Após a prensagem dos painéis, os mesmos foram retirados da prensa e, acondicionados por 72 horas, segundo o especificado pelo documento normativo ABNT NBR 14810-2/2013, até a obtenção dos corpos de prova.

\section{CARACTERIZAÇ̃̃O MECÂNICA DOS PAINÉIS}

Para a realização dos ensaios mecânicos, utilizaram-se os corpos de prova conforme dimensões especificadas pela NBR 14810-2/2013. Os testes aconteceram em uma Máquina Universal de Ensaios, modelo EMIC DL $30000 \mathrm{~N}$.

Os testes mecânicos realizados foram tração perpendicular, conhecida como adesão interna ( 6 corpos de prova para cada tratamento) e flexão estática (6 corpos de prova para cada tratamento, para determinação de Módulo de Ruptura (MOR) e Módulo de Elasticidade (MOE). Não determinou-se um valor de carga para os corpos de prova, a carga foi aplicada até que o corpo de prova fosse rompido. Os dados de carga de ruptura e deformação foram coletados pelo software da máquina de ensaio. Os dados de MOR e MOE foram calculados pela própria máquina, onde os dados necessários foram incluídos.

\section{ANÁLISE ESTATIÍSTICA}

A análise estatística foi realizada no programa de estatística R. Sendo verificada a análise de variância através do teste $\mathrm{F}$ ao nível de $5 \%$ de probabilidade, e, também o teste de Tukey, visando à comparação entre as médias dos tratamentos, ao nível de $5 \%$ de probabilidade. Para evidenciar a diferença existente entre os tratamentos, adotou-se a utilização de letras para a comparação das médias pelo teste de Tukey, onde as médias acompanhadas pela mesma letra em tabela, não diferem estatisticamente entre si, com nível de significância de $5 \%$ de probabilidade

\section{RESULTADOS}

\section{ENSAIOS MECÂNICOS}

Os ensaios mecânicos foram baseados no documento normativo brasileiro para painéis de madeira aglomerada NBR 14810-2/2013. Os dados obtidos na realização dos ensaios mecânicos de adesão interna e flexão estática para a 
determinação de Módulo de Elasticidade e Módulo de Ruptura estão apresentados na (Tabela 2).

Tabela 2-Resultados de ensaios mecânicos para os três tratamentos.

\begin{tabular}{cccc}
\hline Ensaios & Tratamento 1 & Tratamento 2 & Tratamento 3 \\
Adesão Interna (MPa) & $0,48 \mathrm{~A}$ & $0,42 \mathrm{~B}$ & $0,38 \mathrm{~B}$ \\
MOE (MPa) & $1875 \mathrm{~A}$ & $1692 \mathrm{~B}$ & $1387 \mathrm{C}$ \\
MOR (MPa) & $12,35 \mathrm{~A}$ & $10,98 \mathrm{~B}$ & $8,43 \mathrm{C}$ \\
\hline
\end{tabular}

*Médias seguidas pelas mesmas letras não apresentam diferença estatística (Tukey, $\alpha=$ $0,05)$

\section{DISCUSSÃO}

\section{TRAÇÃO PERPENDICULAR}

De acordo com a (Tabela 2) os resultados obtidos no ensaio mecânico de tração perpendicular (adesão interna) permitem concluir que o Tratamento 1 e o Tratamento 2 apresentaram dados superiores ao mínimo estabelecidos pela norma ABNT 14810-2/2013, no entanto, de acordo com a análise estatística o Tratamento 3 não difere do Tratamento 2, podendo ser observado na (Figura 1).

Figura 1 - Análise entre os dados de Adesão Interna

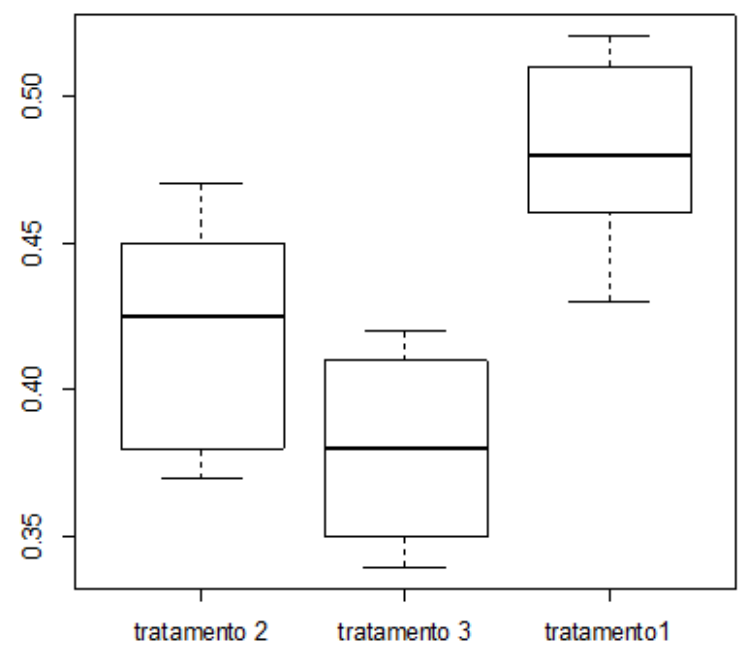

Fonte: Autores (2018)

Com isso, pode-se afirmar que o Tratamento 3 também se encontra de acordo com a norma, se analisado estatisticamente, que especifica para painéis com espessura de 8 a $14 \mathrm{~mm}$ o valor mínimo de 0,40 MPa. Pode-se afirmar ainda segundo a Figura 3 que os dados apresentam grande variabilidade, visto que os valores de mínimo e máximo se encontram bem distantes entre si, justifica-se isso devido à anisotropia do material. Comparando o resultado obtido por Dacosta et al. (2005) que apresentou em seu estudo dados menores que 0,20 $\mathrm{MPa}$, sendo dados inferiores ao apresentado neste presente estudo. 


\section{MÓDULO DE RUPTURA}

Ainda conforme a Tabela 4, os dados obtidos nos ensaios de flexão, para determinação do módulo de ruptura encontra-se inferior ao mínimo especificado pela norma ABNT 14810-2/2013, de $11 \mathrm{Mpa}$, para os Tratamentos 2 e 3. Já o Tratamento 1 apresentou valor superior, indicando condições ideais segundo o requisito de painéis para uso interno em condições secas. Conforme a Figura 4, pode-se afirmar que nos Tratamentos 1 e 2 não houve grande variabilidade de dados, sendo que o Tratamento 3 houve uma dispersão maior. Ainda segundo a (Figura 2), afirma-se que o Tratamento 1apresenta uma distribuição simétrica em relação aos demais Tratamentos.

Figura 2- Análise entre os dados de Módulo de Ruptura

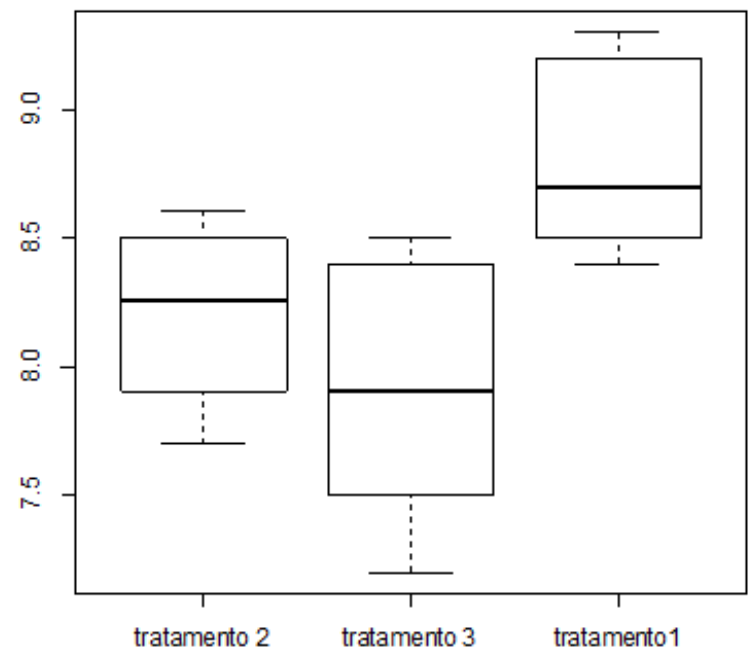

Fonte: Autores (2018)

\section{MÓDULO DE ELASTICIDADE}

Os resultados para Módulo de Elasticidade, o Tratamento 1 obteve valor superior ao especificado pela norma ABNT 14810-2/2013 de $1800 \mathrm{MPa}$, os demais tratamentos apresentaram valor inferior ao estabelecido. A Figura 3 demonstra que os valores obtidos em cada Tratamento diferem significativamente entre si, sendo que apenas o Tratamento 1 atendeu as especificações do valor de referência. 
Figura 3- Análise entre os dados de Módulo de Elasticidade

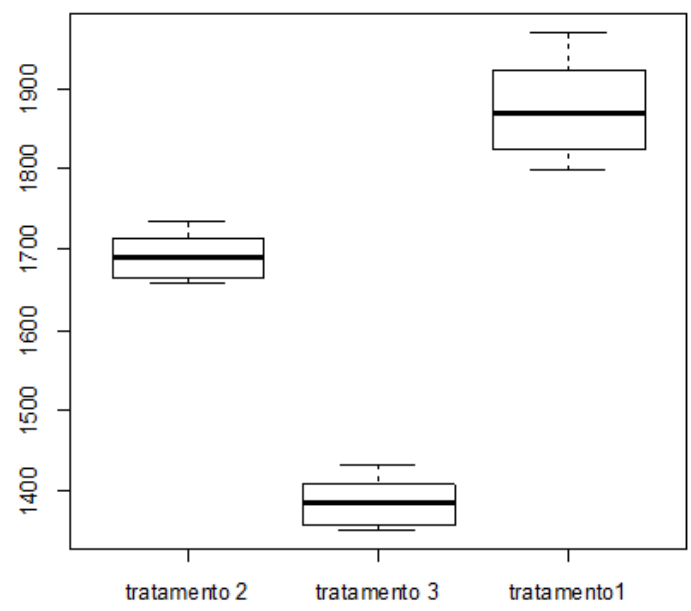

Fonte: Autores (2018)

\section{CONCLUSÃO}

A interação entre as partículas de madeira é decisiva no desempenho final do painel particulado e, dentro deste contexto, pode-se afirmar que a geometria das partículas interfere diretamente nas condições finais do painel particulado de madeira.

Neste estudo pode-se concluir que, o Tratamento 1, produzido com $30 \%$ de partículas de 9 mesh e $70 \%$ de 16 mesh apresentou melhores propriedades mecânicas em relação aos demais tratamentos. As geometrias das partículas influenciaram significativamente nos resultados obtidos, pois no Tratamento 1 havia uma quantidade maior de partículas menores na composição da camada interna. Tal composição proporcionou menor quantidade de vazios na formação do painel que por consequência, melhores propriedades mecânicas. O Tratamento 2, apresentou dados semelhantes ao Tratamento 1, no entanto, alguns ensaios mostraram, que o tratamento 1 ainda é melhor, ou seja, maior quantidade de partículas menores proporcionam melhor interação final do conjunto. E da mesma forma, pode-se analisar o Tratamento 3, onde ocorreu maior incidência de partículas maiores na camada intermediária interferindo de forma negativa no desempenho final do painel.

Levando em consideração que a matéria-prima utilizada na fabricação dos painéis de MDP ser resíduos de madeira de eucalipto, provenientes do processamento mecânico, os resultados obtidos nos ensaios mecânicos mostraram-se aceitáveis quando comparados com o documento normativo, sendo assim viável o uso de resíduos na produção dos painéis.

\section{AGRADECIMENTOS}

Ao PIBIC/CNPq pela concessão de Iniciação Científica sem bolsa. 


\title{
Mechanical characterization of medium density particulate panels produced from wood residue
}

\begin{abstract}
In recent years, the forestry sector has been distinguished by technological innovations and creations of new materials and products. The generation of waste related to wood processing is still a major problem for the segment, because although the new technologies present more modern machinery, the volume generated is still quite significant. With the expressive generation of waste in forest-based industries, the need to use these wastes, in order to add value to them and minimize the environmental impact provided, arises. In this segment of new wood-based materials from waste as source of raw material can be highlighted the panels, mainly, particulate (MDP) and fibers (MDF).The MDP is part of the new generation of Medium Density Particulate Panels; its characteristics are superior and totally distinct from the old agglomerated wood panels. Starting from this disposition of the wood-based products market, the objective of this study was to produce a wood panel from Eucalyptus wastes and assess the behavior of the mechanical resistance against the variation of the granulometric percentage of the particles of the internal layer of this panel. The mechanical characterization was based on the normative document of the Brazilian Association of Technical Standards ABNT-NBR 14810-2 / 2013. The results obtained were statistically analyzed and then compared with the Brazilian standard as well as papers found in the literature.
\end{abstract}

KEYWORDS: MDP. Description. Residue. 


\section{REFERÊNCIAS}

ASSOCIAÇÃO BRASILEIRA DE NORMAS TÉCNICAS. NBR 14810-2: Chapas de madeira aglomerada: requisitos. Rio de Janeiro: ABNT, 2013.

ALMEIDA, Paula Cristina de Oliveira Russo. Estudo da influência da temperatura e do tempo de prensagem nas características físico mecânicas, de diferentes tipos de MDF aquando do seu revestimento por prensagem. Disponível em:<https://www.repository.utl.pt/bitstream/10400.5/1078/1/TESE\%20FINAL\%2 OPDF_\%20Paula\%20Russo.pdf>. Acesso em: 20 jul. 2018.

APRE (Org.). Estudo setorial APRE. Disponível em: $<$ http://www.apreflorestas.com.br/wp-content/uploads/2018/02/EstudoSetorial-2018_APRE.pdf >>. Acesso em: 20 jul. 2018.

BNDES SETORIAL. Painéis de Madeira no Brasil: Panorama e Perspectivas. 27. ed. Rio de Janeiro, 2008. p. 121-156. Disponível em:

http://www.bndes.gov.br/SiteBNDES/export/sites/default/bndes_pt/Galerias /Arquivos/conhecimento/bnset/set2706.pdf. Acesso em: 15 jul. 2018

DACOSTA, L. P. E.; HASELEIN, C. R.; SANTINI, E. J.; SCHNEIDER, P. R.; CALEGARI, L. Propriedades físicas de chapas de partículas aglomeradas fabricadas com resíduos do processamento mecânico da madeira de Pinus elliottii Engelm. Ciência Florestal, Santa Maria, v. 15, n. 4, p. 421-429, 2005. crossref

GONÇALVES, M. T. T. Processamento da Madeira. Bauru. São Paulo. 242p, 2000.

IBÁ (Ed.). MDP - Medium Density Particleboard - Painéis de Partículas de Média Densidade. Disponível em: <http://iba.org/pt/9-conteudo-pt/587-mdp-mediumdensity-particleboard-paineis-de-particulas-de-media-densidade>. Acesso em: 15 jul. 2018

IRLE, M. A.; BARBU, M. C.; REH, R.; BERGLAND, L.; ROWELL, R. M. Wood Composites. In: ROWELL, R. M. Handbook of wood chemistry and wood composites.Boca Raton: CRC Press, 2012. Cap. 10. crossref

IWAKIRI, S. Painéis de madeira reconstituída. Curitiba: FUPEF, 2005. 247 p.

MALONEY, T. M. Modern particleboard \&dry-process fiberboard. 2. ed. San Francisco: Miller Freeman, 1993. 681p. 
PIERRE, F. C. Caracterização físico-mecânica de painéis aglomerados de Eucalyptus grandis com adição de resíduos industriais madeireiros. 2010. 134 p. Tese (Doutorado em Ciência Florestal) - Faculdade de Ciências Agronômicas Campus de Botucatu, Universidade Estadual Paulista "Júlio de Mesquita Filho", Botucatu, São Paulo, 2010.

REMADE 2006 (Ed.). MDP - Nova tendência na fabricação de chapas. Disponível em:

$<$ http://www.remade.com.br/br/revistadamadeira_materia.php?num=978\&subj ect=E mais\&title=MDP- Nova tendencia na fabricao de chapas $>$. Acesso em: 15 jul. 2018.

SOUZA, A.M. ; NASCIMENTO, M.F.; ALMEIDA, D.H.; LOPES SILVA, D.A.; CHRISTOFORO, A.L.; LAHR, F.A.R.Wood-based composite made of wood waste and epoxy based ink-waste as adhesive: A cleaner production alternative. Journal of Cleaner Production, v. 193, p.549-562, 2018. crossref

WEBER, C. Estudo sobre viabilidade de uso de resíduos de compensados, MDF e MDP para produção de painéis aglomerados. 2011.

YOUNGQUIST, J. A - WoodBased composites and panel products - Wood Handbook -Wood as an engineering material - Madison, WI - pg. 10.1-10-31 1999 - USA.

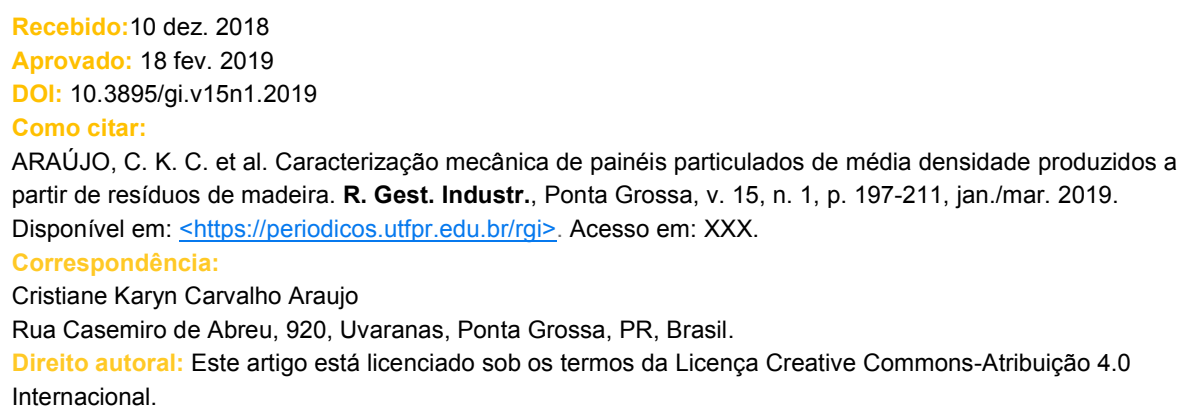

